\title{
Thermodynamics based on the principle of least abbreviated action: entropy production in a network of coupled oscillators
}

\author{
Vladimir García-Morales, ${ }^{1, \circledast}$ Julio Pellicer, ${ }^{2}$ and José A. Manzanares ${ }^{2}$ \\ ${ }^{1}$ Physik-Department E19, Technische Universität München, \\ James-Franck-Str. 1, D-85748 Garching, Germany \\ ${ }^{2}$ Departament de Termodinàmica, Universitat de València, C/Dr. Moliner 50, E-46100 Burjassot, Spain
}

\begin{abstract}
We present some novel thermodynamic ideas based on the Maupertuis principle. By considering Hamiltonians written in terms of appropriate action-angle variables we show that thermal states can be characterized by the action variables and by their evolution in time when the system is nonintegrable. We propose dynamical definitions for the equilibrium temperature and entropy as well as an expression for the nonequilibrium entropy valid for isolated systems with many degrees of freedom. This entropy is shown to increase in the relaxation to equilibrium of macroscopic systems with short-range interactions, which constitutes a dynamical justification of the Second Law of Thermodynamics. Several examples are worked out to show that this formalism yields the right microcanonical (equilibrium) quantities. The relevance of this approach to nonequilibrium situations is illustrated with an application to a network of coupled oscillators (Kuramoto model). We provide an expression for the entropy production in this system finding that its positive value is directly related to dissipation at the steady state in attaining order through synchronization.
\end{abstract}

PACS numbers: 45.50.Jf, 05.70.Ln, 05.45.Xt

\section{INTRODUCTION}

Variational principles occupy a privileged place in the history of physics. They provide approximate results to formidable problems where it is difficult to find analytical solutions from other methods [1]. The first variational principle was established in mechanics by Maupertuis (1744). Its interest and importance was later realized by Lagrange, who referred to it as the most beautiful and important discovery of Mechanics [1]. This principle led to an elegant geometrization of mechanics [2, 3] for conservative systems, and had enormous consequences in the subsequent development of physics.

The variational principles of mechanics constituted the key idea of the former mechanical approaches to thermodynamics. These were first attempted independently by Boltzmann and Clausius and later continued by Helmholtz [4, 5]. These pioneering works allowed for a certain understanding of thermodynamics from mechanics [5, 6] but their importance was later underestimated in favour of a statistical interpretation of thermodynamics. The history of these developments and the confusion surrounding Boltzmann ideas have been recently illuminated by some authors [5, 7, 8, 9, 10, 11].

Despite one century of successful results coming from the statistical approach, the bridge between microscopic dynamics and macroscopic behaviour is still an open issue. Probabilistic concepts are not only extremely useful in practical situations but play also a central role in laying the microscopic foundations of Thermodynamics. The following criticism by Einstein to Boltzmann (who realized the importance of the statistical approach

*Electronic address: vmorales@ph.tum.de in later works) 12 still holds: the systems considered in statistical mechanics are dynamical systems, consisting of moving particles [and therefore] the statistical results should all be derivable from the dynamics.

In this article, we present some novel thermodynamic ideas which are grounded in the Maupertuis principle as well as in the modern general theory of dynamical systems. Ergodicity and probability assumptions (phase space averaging) are not invoked, and only time averages coming from the microscopic mechanics are considered. Our approach makes use of Hamiltonians given in terms of action-angle variables

$$
H=H_{0}(\mathbf{J})+H_{1}(\mathbf{J}, \theta)
$$

where $(\mathbf{J}, \theta) \equiv\left(\left\{J_{i}\right\},\left\{\theta_{i}\right\}\right)$ are vectors containing suitable action and angle variables respectively, and $H_{0}$ and $H_{1}$ are, respectively, the "integrable" and "nonintegrable" parts of the Hamiltonian. It is generally possible to construct such Hamiltonians from, for example, mechanical variational principles 1, 13, 14]. Then, we show that thermal states can be fully characterized by the action variables. When the system is nonintegrable, these variables do not need to be adiabatic invariants but the entropy, defined as a function of them, must not change significantly within a period of the oscillation of any degree of freedom (DOF).

The outline of the paper is as follows. In Section II we introduce the dynamical concepts of equilibrium temperature, entropy and heat differential. The central concept in our approach is the nonequilibrium entropy for a system of many DOFs, which is introduced in Section III. We then link this result to the ones in Section II and a natural, dynamical definition of probability arises, yielding an Einstein relationship at the thermodynamic limit. In Section IV we show that entropy attains its maximum at equilibrium for macroscopic systems with short- 
range interactions. This connects results in Sections II and III. In Section V we apply this dynamically based thermodynamic formalism to several systems of different nature: ideal systems (noninteracting oscillators, ideal gas) and systems with short and long-range interactions. We show how our dynamical definitions correctly reproduce previous equilibrium results obtained from the microcanonical ensemble. Finally, in Section VI, we provide an application to nonequilibrium stationary states in a network of coupled oscillators (Kuramoto model). We study the entropy production in this system by means of the nonequilibrium theory developed in Sections III and IV and illustrate its physical significance. We find that the entropy production at the stationary state is directly related to dissipation in attaining order far from equilibrium through synchronization. Numerical calculations are performed to clarify this result.

\section{EQUILIBRIUM DYNAMICAL TEMPERATURE AND ENTROPY}

In the prehistory of Statistical Mechanics (i.e. before the introduction of any probabilistic concept) Boltzmann provided a mechanical foundation of thermodynamics for monocyclic systems with only one DOF [4, 15]. The constant energy trajectories in phase space of these systems (e.g. a single harmonic oscillator) were bounded, periodic orbits, and Boltzmann defined heat as the energy difference between two orbits. Then he introduced a dynamical temperature and proved that its reciprocal is an integrating factor for the heat differential, which allowed him to identify the entropy of the system. In this article we follow a different path inspired in the Maupertuis principle. Our approach is valid for $f$ DOFs and reduces to Boltzmann's theory for $f=1$.

Consider a system whose Hamiltonian $H(\mathbf{q}, \mathbf{p}, t)$ depends on time $t$ and generalized position coordinates $\mathbf{q}(t)=\left(q_{1}(t), \ldots, q_{f}(t)\right)$ and momenta $\mathbf{p}(t)=$ $\left(p_{1}(t), \ldots, p_{f}(t)\right)=\nabla_{q} \mathcal{A}$, where $f$ is the number of DOFs. The Hamilton-Jacobi equation reads then [2]

$$
\frac{\partial \mathcal{A}(\mathbf{q}, t)}{\partial t}+H(\mathbf{q}, \mathbf{p}, t)=0
$$

where $\mathcal{A}=\int L d t$ is the Lagrangian action and $L$ is the Lagrangian of the system. For conservative systems, $H=$ $E=$ const., action can be varied with respect to the time $\tau$ of the trajectory and the following equation is satisfied [2]

$$
\delta \mathcal{A}+E \delta \tau=0
$$

Since time can be separated in the Hamilton-Jacobi equation, after integration we obtain $\mathcal{A}=J_{E}-E \tau$ where $J_{E}=\int_{\mathbf{q}_{0}}^{\mathbf{q}_{1}} \mathbf{p} d \mathbf{q}$ is the abbreviated action. The Maupertuis principle of classical mechanics states that the $a b$ breviated action $J_{E}$ of the trajectory of the motion is an extremum over all possible energy-conserving trajectories that pass through ending points $\boldsymbol{q}_{0}$ and $\boldsymbol{q}_{1}$ in an arbitrary time $\tau$ [2].

From the total variation of the action $\delta \mathcal{A}=\frac{\partial J_{E}}{\partial E} \delta E-$ $\tau \delta E-E \delta \tau$, and Eq. (3), we have

$$
\frac{\partial J_{E}}{\partial E}=\tau
$$

In the case of closed orbits this equation is written as

$$
\frac{\partial J_{c}}{\partial E}=\tau_{c}
$$

where $J_{c}=\oint \mathbf{p} d \mathbf{q}$ is the abbreviated action of a closed orbit and $\tau_{c}$ is the (Poincaré) time needed for its completion. It is then clear that the product of $J_{c}$ and the recurrence frequency $\omega_{c} \equiv 1 / \tau_{c}$ has dimensions of energy. We define the dynamical equilibrium temperature as

$$
T^{(e q)} \equiv \frac{\omega_{c} J_{c}}{f k}
$$

where $k$ is the Boltzmann constant. A similar definition was introduced by Boltzmann for the case $f=1$ (see [4], p. 419). Since $J_{c}$ is an invariant of the motion and does not depend on the choice of coordinates [2], the equilibrium temperature does not depend on this choice either.

The dynamical definition of the equilibrium entropy $S^{(e q)}$ is introduced so that the thermodynamic equation $\partial S^{(e q)} / \partial E=1 / T^{(e q)}$ is satisfied. Since Eqs.(5) and Eq. (6) lead to

$$
f k \frac{\partial \ln J_{c}}{\partial E}=\frac{1}{T^{(e q)}}
$$

the equilibrium entropy $S^{(e q)}$ is defined as

$$
S^{(e q)} \equiv f k \ln \left(a J_{c}\right)
$$

where $a$ is an integration constant with dimensions of inverse action (in the following we take $a$ as unity). Consistently, the heat differential is defined as

$$
d Q \equiv \omega_{c} d J_{c}
$$

which represents the variation of energy involved in changing the action on the closed trajectory. Thus, it is satisfied that $1 / T^{(e q)}$ is an integrating factor for the heat differential, $d Q=T^{(e q)} d S^{(e q)}$.

These thermodynamic expressions are useful if we are able to calculate $J_{c}$ from the microscopic dynamics. For systems with $f=1$ and bounded orbits this is always possible since the Hamiltonian is integrable. The constant energy $E$ can then be directly related to $J_{c}$. For systems with many DOFs, ergodic theory has provided the means for calculating $J_{c}$. This theory establishes the equality between time averages and phase space averages under certain conditions and the Birkhoff theorem. Our approach does not require ergodicity but leads to the same results if Birkhoff theorem holds, as shown in Appendix A. 


\section{NONEQUILIBRIUM ENTROPY}

To extend the formalism to nonequilibrium systems with many DOFs, we write the Hamiltonian as in Eq. (1) by means of an appropriate canonical transformation. This yields a set of action-angle variables $(\mathbf{J}, \theta)$ whose evolution is then considered. The term $H_{1}$ in Eq. (1) does not come from a perturbation expansion and it is not necessarily smaller than $H_{0} . H_{1}$ is associated to energy exchange between DOFs in the form of heat. In that case the $J_{i}$ 's are neither adiabatic invariants nor constants of the motion but suitable generalized coordinates with dimensions of action. These action-angle variables are to be understood as effective ones, coming from approximations consistent with the dynamics of the Hamiltonian under consideration (a criterion to be satisfied for appropriate action variables is given below). Since each DOF oscillates periodically with its effective frequency, it is physically meaningful to consider the action $J_{i}$ of the DOF $i$ on its closed cycle with the understanding that this cycle can be completed by the DOF going back and forth following the Hill's region (i.e. the region in configuration space to which the DOF is confined) until it reaches its initial angle. Only in the limit of vanishing $H_{1}$ are the action variables adiabatic invariants also: they correspond to the integrable part of the Hamiltonian and solve exactly the integrable dynamics. This kind of representations is used in plasma physics and can be constructed for general Hamiltonians [16].

We define the "temperature" and "entropy" of the DOF $i$ as

$$
\begin{gathered}
T_{i} \equiv \frac{\omega_{i} J_{i}}{k} \\
S_{i} \equiv k \ln J_{i}
\end{gathered}
$$

so that the thermodynamic formalism for one DOF resembles that for the whole system. The energy of each DOF $\varepsilon_{i}(i=1, \ldots, f)$ can vary subject to the constraint that the total energy $E=\sum_{i=1}^{f} \varepsilon_{i}$ is constant, and it is satisfied that $\partial S_{i} / \partial \varepsilon_{i}=1 / T_{i}$. These definitions are reasonable because Eqs. (6) and (8) apply to any mechanical system, regardless of its size, and each DOF in the Hamiltonian behaves as a "system" with energy $\varepsilon_{i}$. However, contrarily to the many-DOF system for which the total energy $E$ is a constant, each DOF usually exchanges energy with the others (provided that $H_{1}$ in Eq.(11) is nonvanishing and the DOFs are separated in $\left.H_{0}\right)$. The energy $\varepsilon_{i}$ is to be understood as a time average on the Hill's region in which the DOF moves. This is clear from the Hamilton-Jacobi equation, which can be applied both to the composite system and to each DOF separately with the understanding that, in the latter case, each DOF behaves as a system exchanging energy with the others and its energy is, therefore, a time average.

The entropy of one DOF, Eq. (11) can of course change with time but to be physically meaningful it must not change significantly during one period $\tau_{i}=1 / \omega_{i}$ of the oscilation of the DOF i. The DOF $i$ has explored its whole available Hill region only after completing a period. The entropy of one DOF is defined in terms of the whole available Hill region at a given time $t$ and it would be inconsistent not to have an almost constant value of this quantity within one period of the oscillation. This leads to stablish a criterion on the suitability of the action variables which, as pointed out above, need not to be adiabatic invariants. The condition to be satisfied comes from expanding the entropy of a DOF $S_{i}$ in a time $t+\tau_{i}$ respect to its value at time $t$. Keeping only linear terms in $\tau_{i}$ we have

$$
\frac{S_{i}\left(t+\tau_{i}\right)}{S_{i}(t)} \approx 1+\frac{\tau_{i}}{S_{i}(t)} \frac{d S_{i}(t)}{d t}
$$

The action variables are appropriate when the absolute value of the second term in the r.h.s of Eq. (12) is much lower than unity. This implies

$$
\left|\frac{1}{\omega_{i}} \frac{d}{d t} \ln \ln J_{i}\right|<<1
$$

If the action variables $J_{i}$ are adiabatic invariants this criterion is automatically (and in the best way) satisfied. But this is only a sufficient condition and not a necessary one. Given any Hamiltonian in action-angle variables, the criterion in Eq. (13) can be applied to decide if the action variables are appropriate or if a canonical transformation to find a better set of variables is needed. If this condition is met, the temperature of one DOF is also well defined from Eq. (10)

$$
\frac{1}{k T_{i}}=\frac{\partial \ln J_{i}}{\partial H}
$$

It is to be noted that action variables bear geometric properties of the phase space in the large [17]. This can be seen in the fact that in obtaining the action variable $J_{i} \equiv \oint p_{i} d q_{i}$ the momentum of the $i$-th DOF $p_{i}$ is integrated on a closed domain in configuration space involving the coordinate position $q_{i}$. This closed domain (a 'monocycle') in $q_{i}$ is the definition of Hill's region of DOF $i$. The Hill's region can be a macroscopic object and, therefore, action variables connect in this way the micro- and macroscales: The former one is suggested by the fact that we address each DOF separately; the latter comes in the distance over which the DOF moves, an information contained in its action variable. In a complicated problem, any assumption made in approximating action variables is connected to an assumption on how the trajectory fills the available phase space. This is of special relevance for the Second Law (discussed in the next section) to hold since a nonvanishing $H_{1}$ in Eq. (11) is then required. This in turn implies that the action-angle variables considered are already 'effective' i.e. 'approximate' ones (the exact action-angle variables would make the Hamiltonian independent of angle variables, the system being integrable). A nonvanishing $H_{1}$ in the actionangle representation is necessary to get relaxation to 
equilibrium coming from the thermalization of the DOFs. This term exist because of, for example, having interactions between many DOFs that make the system nonintegrable. The coarse-graining procedure as employed in the foundations of standard Statistical Mechanics can be also thought as contained in a particular assumption made on the action variables: the one sketched in Appendix A in which every possible point in phase space is equally visited and distributed over the Hill's regions of all DOFs. Under such an approximation the dynamical trajectory is a one-cycle permutation of all attainable points (or 'microstates') in phase space. This allows to speak about a phase space volume instead of a dynamical trajectory and to introduce well-behaved probability distributions related to 'densities' of occupancy of the phase space. In [18] this and other assumptions leading to different Statistical Mechanics formalisms are discussed from a different (but closely related) viewpoint. Common to both lines of reasoning in [18] and here is that for the possibility of providing sound equilibrium statistical arguments motivated by the dynamics, the system trajectory should be long enough in order to approximate the volume of phase space in which it is contained.

A situation of upmost interest occurs when the total energy is equally shared by all DOFs of the system, so that $\varepsilon_{i}=\varepsilon^{(e q)} \equiv E / f$ for every DOF. In this case, the temperature of every DOF is equal to the equilibrium temperature of the system defined by Eq.(6),$T_{i}=T^{(e q)}$. This equilibrium state of the system is characterized by the conditions $T_{i}=T^{(e q)}$ and $J_{c} \omega_{c}$ finite. In relation to the latter condition, it must be noticed that $\omega_{c}$ can be vanishingly small for macroscopic systems but then $J_{c}$ is large. Thus, in an equilibrium state it is satisfied that $J_{c} \omega_{c}=f k T^{(e q)}=\sum_{i=1}^{f} J_{i} \omega_{i}$ and therefore

$$
f k T^{(e q)}=\sum_{i=1}^{f} J_{i} \frac{\partial E}{\partial J_{i}}=k \sum_{i=1}^{f} \frac{\partial E}{\partial S_{i}}
$$

This provides the energetic equation of the system.

In a nonequilibrium state, however, the temperatures and energies of each DOF are different. The nonequilibrium entropy of the system in such cases is

$$
S \equiv \sum_{i=1}^{f} S_{i}=k \ln \prod_{i=1}^{f} J_{i}=f k \ln J_{E}^{*}
$$

It depends on each $\varepsilon_{i}$ through the action variables. This entropy corresponds to an open segment on the closed trajectory with abbreviated action $J_{E}^{*} \equiv\left(\prod_{i=1}^{f} J_{i}\right)^{1 / f}$, and the last equality in Eq. (16) makes this explicit. It is worth noting that Eqs.(11) and (16) coincide with Eq. (8) when the system has only one DOF. The same applies to temperatures defined by Eqs. (6) and (10).

For a closed trajectory it is satisfied that

$$
\omega_{c} J_{c}=\omega_{c} \int_{0}^{\tau_{c}} \mathbf{p} \dot{\mathbf{q}} d t=<2 K>_{\tau_{c}}
$$

where $K$ is the kinetic energy. (It may be remembered that the condition $\delta J_{E}=0$ leads to the equation of the geodesics of the Jacobi metric $g_{i j}=2 K \delta_{i j}[3]$.) A segment of the closed trajectory is said to be typical if

$$
<2 K>_{\tau}=\frac{1}{\tau} \int_{t_{1}}^{t_{1}+\tau} \mathbf{p} \dot{\mathbf{q}} d t=<2 K>_{\tau_{c}}=f k T^{(e q)}
$$

where $\tau$ is the duration of the segment (in all what follows $\tau$ must be understood as a much longer time than the period $\tau_{i}$ of the slowest DOF $i$ ). Therefore, the nonequilibrium entropy for a typical segment is equal to the equilibrium entropy, and this opens the possibility of defining the equilibrium thermodynamic variables in terms of the action over a typical segment rather than in terms of the action over the whole closed (macro)trajectory, as done in the previous section. That is, we can consider that at equilibrium the system moves along typical segments. These considerations are further explored in Appendix B. In relation to this, it is remarkable that the existence of an equilibrium state implies, from Eqs. (86) and (16)

$$
\lim _{t \rightarrow \tau_{r}<\tau_{c}}\left[\prod_{i=1}^{f} J_{i}\left(\varepsilon_{i}\right)\right]=\left[J_{c}(E)\right]^{f}
$$

where $\tau_{r}$ is the relaxation time. That is, if the system has a state of thermodynamic equilibrium it attains this state after a time $\tau_{r}$ lower than $\tau_{c}$. This suggests that the lower is $\tau_{r}$ (compared to $\tau_{c}$ ) the more accurate is the description of the equilibrium state in terms of typical segments. This is because departures from equilibrium are then short and, therefore, the closed trajectory contains a higher number of typical segments.

Since we can use Eq.(16) also to deal with equilibrium situations provided we address typical segments, action variables illuminate first-order equilibrium phase transitions involving a discontinuous change in the entropy. If we think on a Lennard-Jones fluid, for example, the Hill's region of a DOF of a bound particle changes discontinuously when changing (continuously) the total energy of the particle from $E<0$ to $E>0$. In the former situation, the Hill's region is bounded by the attractive region of the potential. In the latter one, the Hill's region is bounded by the macroscopic volume where the fluid is contained. This signals the transition from a liquid to a gas state, involving a discontinuity on the value of the action variables of many DOFs (since their energy is varied continuosly but their Hill's regions change abruptly) and, therefore, a discontinuity on the entropy, Eq. (16).

The probability $P(\gamma)$ that the system can be found in a given segment $\gamma$ [i.e. a piece of micro-trajectory on the closed (macro)trajectory] is given by the ratio between its duration $\tau$ and the total duration of the closed trajectory $\tau_{c}$

$$
P(\gamma)=\frac{\tau}{\tau_{c}}=\frac{\partial J_{E}^{*} / \partial E}{\partial J_{c} / \partial E}=\frac{1}{f k} \frac{\partial S(\gamma) / \partial E}{\partial J_{c} / \partial E} e^{S(\gamma) / f k}
$$

where $S$ is the nonequilibrium entropy in the segment. If we ask for the probability of measuring certain macrovariables (as $T^{e q}$ ) during a finite measurement time, it is clear 
from the above that this is given by $P\left(\gamma_{1} \bigcup \gamma_{2} \ldots \bigcup \gamma_{n}\right)=$ $P\left(\gamma_{1}\right)+P\left(\gamma_{2}\right)+\ldots P\left(\gamma_{n}\right)$ where the $\gamma_{i}$ 's are all segments on the closed trajectory consistent with the macrostate characterized by the macrovariables whose probability we ask. Two consecutive typical segments of durations $\tau_{1}$ and $\tau_{2}$ produce a typical segment of duration $\tau_{1}+\tau_{2}$. However, the contrary is not necessarily true: not all pieces on an arbitrary decomposition of a typical segment are also, in general, typical ones.

Eq.(16) suggests a roughly linear dependence of $S$ on $f$ and, in the thermodynamic limit, Eq. (20) can be simplified to

$$
P(\gamma) \approx c e^{S(\gamma) / f k}
$$

where $c$ is a constant. This equation states that segments with the highest entropy are also the most probable ones. These segments are also the typical ones since, on them, the average in Eq. (18) coincide with the average over the whole trajectory. This is a result of the mean value theorem for integrals. Note, finally, the similarity of Eq. (21) and Einstein's inversion formula [19]. The latter equation is the basis for Gibbs' variational principle (maximum entropy approach) that allows to develop equilibrium statistical thermodynamics.

\section{THE SECOND LAW IN MACROSCOPIC SYSTEMS WITH SHORT-RANGE FORCES}

In most macroscopic systems with short-range interactions the relation between the energy and the action for every DOF is $J_{i}=c_{i} \varepsilon_{i}^{p}$ where $p$ is a positive exponent (and $c_{i}$ does not depend on energy) and the entropy is then

$$
S=k \ln \prod_{i=1}^{f} c_{i}+k p \ln \prod_{i=1}^{f} \varepsilon_{i}
$$

At equilibrium, $\varepsilon_{i}=\varepsilon^{(e q)} \equiv E / f, E=p f k T^{(e q)}$ from Eq. (15), and

$$
S^{(e q)}=k \ln \prod_{i=1}^{f} c_{i}+f k p \ln \varepsilon^{(e q)}
$$

We can use the following mathematical inequality [20] (the geometric mean of a series of values $\varepsilon_{i}$ is lower than its arithmetic mean)

$$
\left(\prod_{i=1}^{f} \varepsilon_{i}\right)^{1 / f} \leq \frac{1}{f} \sum_{i=1}^{f} \varepsilon_{i}=\varepsilon^{(e q)}
$$

then, since $p$ is positive, by comparing Eqs.(22) and (23) we obtain that

$$
S^{(e q)} \geq S
$$

where the equality only holds at equilibrium. Therefore, in the evolution to equilibrium $\Delta S \geq 0$. This constitutes the mathematical statement of the second law for conservative macroscopic systems with short-range interactions.

Thermalization results from the coupling between DOFs and leads to the homogeneity required for all thermal properties at equilibrium. Obviously, no such coupling is possible in a conservative system with $f=1$, and this is always "at equilibrium". During the relaxation to equilibrium, each $J_{i}, \varepsilon_{i}, S_{i}$ and $S$ can change with time. Whether a system has a state of thermodynamic equilibrium or not is determined by the form of the nonintegrable part $H_{1}(\mathbf{J}, \theta)$ of the Hamiltonian in Eq. (1). If it vanishes and the DOFs are separated in $H_{0}$, the system does not have a thermodynamic equilibrium state. The dynamical equations are then integrable with $f$ integral invariants $J_{i}$ and the DOFs are not able to exchange energy because of the existence of regular orbits. In such case the closed trajectory is then only composed of nontypical segments. Nonintegrability of the Hamiltonian is thus necessary to achieve thermodynamic equilibrium.

As the system approaches equilibrium the segments gradually become typical ones. This process is characterized by the entropy production $\sigma$

$$
\sigma \equiv \frac{d S}{d t}=k \frac{d \ln \left(\prod_{i=1}^{f} J_{i}\right)}{d t}=-k \sum_{i=1}^{f} \frac{1}{J_{i}} \frac{\partial H_{1}}{\partial \theta_{i}}
$$

where Eq. (1) and the Hamilton equations of motion for the action variables have been used. At equilibrium $S$ becomes $S^{(e q)}$ which does not depend on time, and the entropy production vanishes.

Relaxation to equilibrium is described most easily through a term $H_{1}=\sum_{i=1}^{f}\left(J_{i}-J_{i}^{(e q)}\right) \theta_{i} / \tau_{r}$ in Eq.(1), with $J_{i}^{(e q)} \equiv J_{i}\left(\varepsilon^{(e q)}\right)$. This term can be thought to appear as a consequence of collisions (or other complex interactions) that thermalize the system. The Hamilton equation $\dot{J}_{i}=-\partial H_{1} / \partial \theta_{i}=-\left(J_{i}-J_{i}^{(e q)}\right) / \tau_{r}$, can be integrated as $J_{i}(t)=\left(J_{i 0}-J_{i}^{(e q)}\right) e^{-t / \tau_{r}}+J_{i}^{(e q)}$. From $J_{i}=c_{i} \varepsilon_{i}^{p}$ we have that the energy $\varepsilon_{i}$ relaxes to $\varepsilon^{(e q)}$ when $J_{i}$ relaxes to $J_{i}^{(e q)}$, and $S$ relaxes then to $S^{(e q)}$. The entropy production for this $H_{1}$ can be calculated by means of Eq. (26) as $\sigma=\frac{k}{\tau_{r}} e^{-t / \tau_{r}} \sum_{i=1}^{f}\left(J_{i}^{(e q)}-J_{i 0}\right) / J_{i}(t)$. This is a positive definite function which vanishes when equilibrium is attained.

Although relaxation processes can be much more complex, action-orbit coupling suffices to describe thermalization in the approach to equilibrium. A relaxation process to a nonequilibrium steady state is illustrated in section VI

\section{EXAMPLES}

In this section we consider Hamiltonians which are either integrable or made integrable through suitable ap- 
proximations based on the dynamics (e.g. the globally chaotic system). When equilibrium quantities are mentioned it should be understood that there is an additional mechanism in the Hamiltonian producing relaxation to equilibrium analogous to the one described in the previous section. Here we are only concerned with the integrable part of the Hamiltonian, from which the relevant action-angle variables are obtained.

\section{A. Noninteracting oscillators}

Consider a system of $N$ noninteracting harmonic oscillators $(f=N)$ with Hamiltonian $H=$ $\sum_{i=1}^{N}\left(p_{i}^{2} / 2 m+m \omega_{i}^{2} q_{i}^{2} / 2\right)$, the $\varepsilon_{i}$ are related to the amplitude $A_{i}$ of each oscillator as $\varepsilon_{i}=m \omega_{i}^{2} A_{i}^{2} / 2$. The energies $\varepsilon_{i}$ depend on the initial conditions in which the system is prepared. The action variables are $J_{i}=$ $\varepsilon_{i} / \omega_{i}$ and hence $p=1$. Eq. (16) provides the entropy as $S=k \ln \left(\prod_{i=1}^{N} \varepsilon_{i} / \omega_{i}\right)$. The equilibrium entropy is $S^{(e q)}=k \ln \left(\prod_{i=1}^{N} \varepsilon^{(e q)} / \omega_{i}\right)$ and the total energy is given by Eq. (15) as $E=N k T^{(e q)}$.

\section{B. Classical ideal gas}

The Hamiltonian for a classical ideal gas of $N$ particles $(f=3 N)$ in a cubic box of volume $V=L^{3}$ closed by adiabatic, rigid walls is

$$
H=\sum_{i=1}^{3 N}\left[\frac{p_{i}^{2}}{2 m}+C \Theta\left(\left|\frac{2 q_{i}}{L}\right|-1\right)\right]
$$

where $C$ is an infinitely large constant and $\Theta$ is the Heaviside step-function. The Hill's region for each DOF is just a segment with length $L$ and the action variables are $J_{i}=2 L \sqrt{2 m \varepsilon_{i}}$, so that $p=1 / 2$. The entropy can be easily calculated and the equilibrium entropy is obtained from it as $S^{(e q)}=k \ln \left[V^{N}(2 m E / 3 N)^{3 N / 2}\right]$ which coincides with the microcanonical one, except for an undetermined constant $a$ like in Eq. (8). From Eq.(15) the energy is $E=3 N k T^{(e q)} / 2$.

We can group the $J_{i}$ appearing in the entropy in blocks of three factors corresponding to each particle. Permutations of the blocks cannot be distinguished and, since there are $N$ ! of these permutations the constant $a$ should be $\propto 1 / N$ !. These considerations constitute the explanation of the Gibbs' paradox. The dependence of the equilibrium entropy on extensive variables $(N, V, E)$ match the traditional calculations and other thermodynamic variables such as pressure and chemical potential can be defined from this entropy [18].

\section{Planetary system}

Let us consider now a system of $N$ particles of mass $m$ moving in closed orbits on the same plane subject to a gravitational potential $(f=2 N)$. Neglecting the interparticle interactions, the Hamiltonian is

$$
H=\sum_{j}^{N}\left[\frac{1}{2 m}\left(p_{r, j}^{2}+\frac{p_{\theta, j}^{2}}{r_{j}^{2}}\right)-\frac{\alpha}{r_{j}}\right]
$$

where subindex $p_{r, j}, p_{\theta, j}$ and $r_{j}$ are the radial and angular momenta and the position of the particle $j$, respectively. Each particle has two DOFs of degenerate frequencies and their energies cannot be separated. As a result, the product of actions in Eq.(16) run now over particles instead of DOFs. The action per particle is $J_{j}=J_{r, j}+J_{\theta, j}=\alpha \sqrt{\frac{m}{2|\varepsilon|_{j}}}[2]$. In this case $p=-1 / 2$, the entropy is $S=2 k \ln \left[\prod_{j=1}^{N} \alpha \sqrt{\frac{m}{2\left|\varepsilon_{j}\right|}}\right]$ and the equilibrium entropy $S^{(e q)}=2 N k \ln \left[\alpha \sqrt{\frac{N m}{2|E|}}\right]$. It is to be noted that this equilibrium is unstable. The energy is $E=-N k T^{(e q)}$ and the heat capacity is negative, $C=-N k$. Negative heat capacities arise frequently in bound gravitational systems [10].

\section{Globally chaotic system in the adiabatic approximation}

Let us consider next a system with $f=2$ and $H=$ $\left(p_{x}^{2}+p_{y}^{2}+x^{2} y^{2}\right) / 2$. It constitutes a simple model of the classical Yang-Mills field. The energies $\varepsilon_{i}$ are not separated in the Hamiltonian due to the coupling term $x^{2} y^{2} / 2$. However, the Hamiltonian can be calculated in action-angle variables by means of the adiabatic approximation as $H=\left(\frac{3 \pi}{4 \sqrt{2}}\right)^{2 / 3} J_{x}^{2 / 3} J_{y}^{2 / 3}[21]$. The entropy is then $S=S^{e q}=k \ln \left[\left(\frac{4 \sqrt{2}}{3 \pi}\right) E^{3 / 2}\right]$ and the energy is $E=3 k T^{(e q)} / 2$. Hence, although the Hamiltonian of this globally chaotic system is complex, the adiabatic approximation based on the dynamics [21] allows us to write it as in Eq. (1). Moreover, the coupling $J_{x}^{2 / 3} J_{y}^{2 / 3}$ between the action variables makes the system to be already thermalized (action variables are not separated in the Hamiltonian) so that under the adiabatic approximation the system is always "at equilibrium".

\section{E. Calogero Hamiltonians with short range forces}

Finally, we consider a system of $N$ particles in one dimension $(f=N)$ with nearest-neighbor interactions, 
periodic boundary conditions and Hamiltonian

$$
\begin{aligned}
H= & \quad N \lambda \sum_{i}^{N} p_{i}+\mu \sum_{i=0}^{N-1} \sqrt{p_{i} p_{i+1}} \cos \left[\nu\left(q_{i}-q_{i+1}\right)\right] \\
& +\mu \sigma \sqrt{p_{i} p_{i+1}} \cos \left[\nu\left(q_{1}-q_{N}\right)\right]
\end{aligned}
$$

This Hamiltonian belongs to a class of fully integrable Calogero Hamiltonians and can be written in actionangle variables as $H=\sum_{i}^{N} J_{i} \omega_{i}$ where the $\omega_{i}$ 's are the eigenvalues of the matrix [22]

$$
C=\left(\begin{array}{ccccc}
0 & 1 & \cdot & \cdot & \sigma \\
1 & 0 & 1 & . & \cdot \\
\cdot & 1 & 0 & 1 & . \\
\cdot & \cdot & 1 & 0 & 1 \\
\sigma & \cdot & \cdot & 1 & 0
\end{array}\right)
$$

where a dot stands for 0 . If $C$ is any hermitian matrix, one can construct a wide class of interesting Hamiltonians that can be used to model many-particle interacting systems with short- and long-range forces [22]. The entropy, equilibrium entropy and energy, $S=k \ln \left(\prod_{i}^{N} \varepsilon_{i=1} / \omega_{i}\right)$, $S^{(e q)}=k \ln \left[(E / N)^{N} \prod_{i=1}^{N} 1 / \omega_{i}\right]$ and $E=N k T^{(e q)}$, coincide with that of $N$ noninteracting harmonic oscillators with frequencies $\omega_{i}$.

\section{NONEQUILIBRIUM THERMODYNAMICS: ENTROPY PRODUCTION IN A NETWORK OF COUPLED OSCILLATORS}

The Kuramoto model [23] (see also [24] and 25] for excellent reviews) has received great recent interest because it provides a simple model for synchronization. This model considers a network of oscillators which are coupled harmonically in phase. After a transient, and under certain conditions as explained below, a fraction of the oscillators in the network become synchronized. This means that both the effective frequency of the oscillators and the phase becomes equal, in spite of the fact that they have different natural frequencies and they start with very different phases [24].

The Kuramoto model is a particular case of the socalled phase models [26, 27] in which angles are coupled through a harmonic function. Every dynamics yielding oscillations to a limit cycle can be transformed in a phase model by means, for example, of the Hilbert transform [26]. Phase models are naturally linked to action-angle descriptions: the phase of each oscillator runs periodically on the limit cycle confining the trajectory, and in completing each cycle a mechanical action (which is conjugate to the phase) is carried out by the system. In our view, the action variables control the thermodynamic properties of the system through Eq. (16) and this opens the possibility of studying these properties using our formalism.
The action-angle Hamiltonian yielding the Kuramoto dynamics is

$$
H=\sum_{i}^{N} J_{i} \omega_{i}^{\circ}+K \sum_{j \neq i}^{N} \sum_{i}^{N} J_{i} \sin \left(\theta_{j}-\theta_{i}\right)
$$

The first term on the r.h.s. describes the uncoupled system of oscillators with natural frequencies $\omega_{i}^{\circ}$ while the second depends on a global coupling parameter $K$ that can be arbitrarily large. The system is nonintegrable because of the explicit appearance of the angle variables in the second term. This makes every action variable $J_{i}$ to evolve in time. The Hamilton equations of motion are

$$
\begin{aligned}
& \omega_{i}=\frac{\partial H}{\partial J_{i}}=\omega_{i}^{\circ}+K \sum_{j \neq i}^{N} \sin \left(\theta_{j}-\theta_{i}\right) \\
& \dot{J}_{i}=-\frac{\partial H}{\partial \theta_{i}}=K J_{i} \sum_{j \neq i}^{N} \cos \left(\theta_{j}-\theta_{i}\right)
\end{aligned}
$$

Eqs. (32) constitutes the Kuramoto dynamics. It establishes the effective frequency of the $i$-th oscillator, which is driven in phase through the all-to-all coupling. In the limit of vanishing coupling $K$ each DOF oscillates with its natural frequency and the oscillators are not synchronized. For $K$ higher than a critical value $K_{c}$, however, some oscillators become synchronized after a transient. In the limit of strong coupling all oscillators are synchronized. This situation is depicted in Fig [1, which has been obtained by numerical integration of Eqs. (32) by employing a fourth order Runge-Kutta algorithm for $N=100$ oscillators starting at random initial phases and with a uniform distribution of natural frequencies in the range $(0, \mathrm{~N}] \mathrm{rad} / \mathrm{s}$. The phases of the oscillators converge into two branches with the same effective frequency and phase. The suitability of the action-angle variables for the parameters considered was confirmed in the simulations by checking Eq. (13) at each time.

Eqs. (33) represent the dynamics of the conjugate action variables and the entropy production can be calculated from Eq.(26) as

$$
\frac{\sigma}{k}=K \sum_{j \neq i}^{N} \sum_{i}^{N} \cos \left(\theta_{j}-\theta_{i}\right)
$$

Remarkably, this equation does not depend on the $J_{i}$ and $\sigma$ can be evaluated after numerical integration of Eqs.(32). If we consider a high number of oscillators, the r.h.s of Eq. (34) vanishes when these are not synchronized, since the phases are distributed uniformly. In the limit of high coupling all oscillators are synchronized, the phases of some oscillators become equal and the r.h.s of Eq. (34) becomes approximately $\sim K N^{2}$ attaining a finite and positive value. This positive sign of the entropy production for networks with many oscillators and long times is perfectly consistent with the Second Law of Thermodynamics. In Fig. 2 we show the value of the normalized 


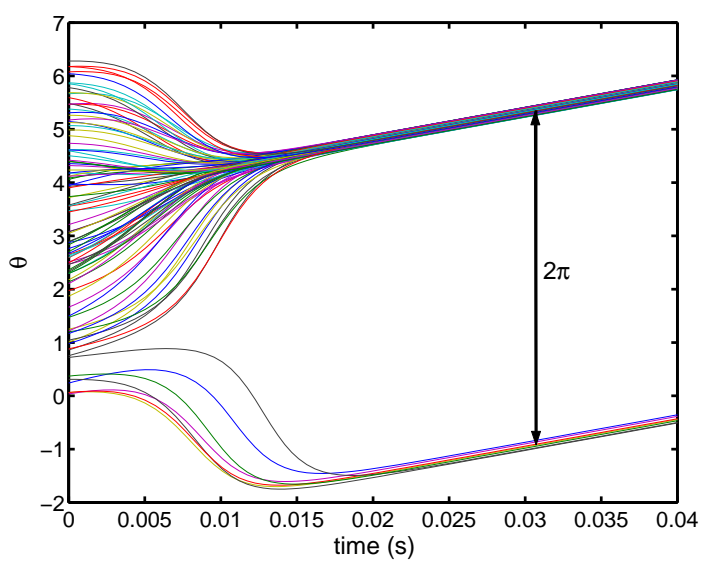

FIG. 1: Time evolution of the phases of 100 oscillators for strong coupling $\mathrm{K}=5$ obtained by numerical integration of Eqs. (32). The system starts with a random distribution of phases and after a short transient all oscillators become synchronized with the emergence of two branches with the same effective frequency.

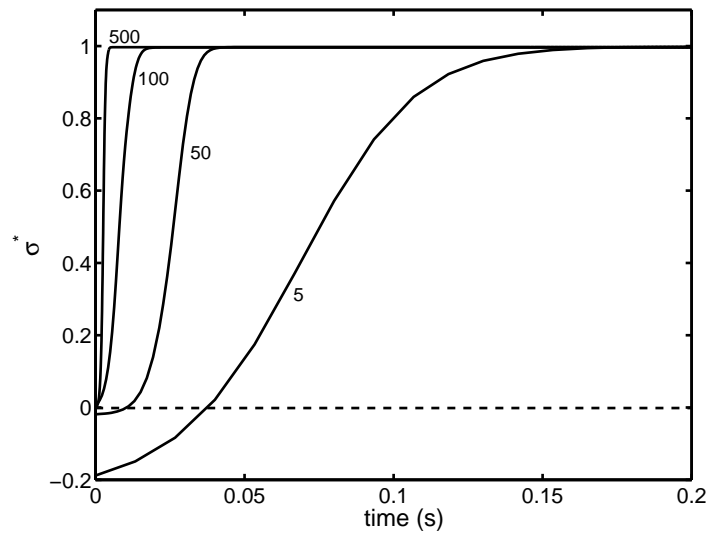

FIG. 2: Time evolution of the normalized entropy production $\sigma^{*}=\sigma /[k K N(N-1)]$ for different networks of oscillators with the values of $N$ indicated in the figure and coupling parameter $K=5$. The system starts with a random distribution of phases and after a short transient all oscillators become synchronized.

entropy production $\sigma^{*} \equiv \sigma /[k K N(N-1)]$ for different network sizes and in the strong coupling limit $(K=5)$. The initial phases are chosen at random. It is observed that in the nonequilibrium steady state, when the oscillators become synchronized, this entropy production is positive and finite in all cases. For networks with high numbers of oscillators, the entropy production is positive at all times in all trajectories starting at random phases. For low number of oscillators and small time scales (far from the thermodynamic limit), negative entropy production can be observed.

Another interesting issue concerns the characterization of disorder. The nonequilibrium entropy calculated

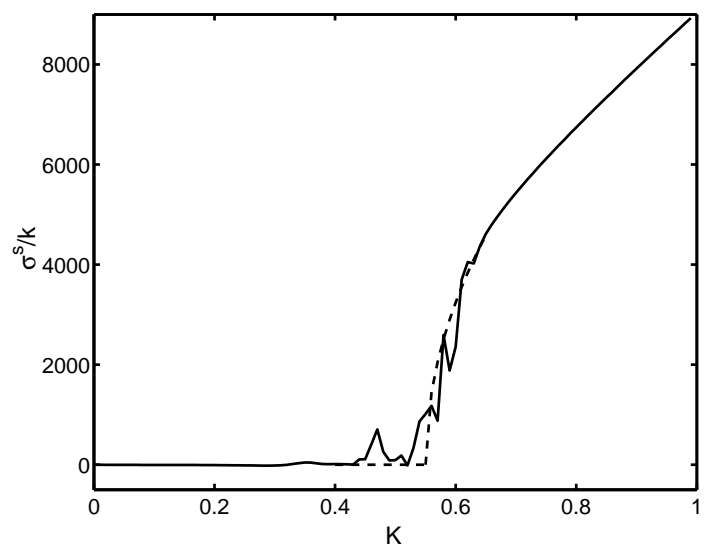

FIG. 3: Entropy production in the long time limit as a function of the coupling parameter for a network of $N=100$ oscillators. The entropy production vanishes for coupling parameter lower than a critical value around $K=0.55$. At the onset of synchronization, the entropy production becomes positive and finite. In the limit of strong coupling, all oscillators are synchronized and the entropy production approaches a line with slope $\sim N^{2}$.

above is directly related to the activity of the network of oscillators and acts itself as an order parameter of the system. In Fig. (3) we show the value of the entropy production at the steady state $\sigma^{s} \equiv \sigma(t \rightarrow \infty)$ as a function of the coupling parameter for a network of $N=100$ oscillators. Quite interestingly, the entropy production is zero for low values of the coupling parameter in which the oscillators are not synchronized. Then, at a critical value $K_{c}$ around 0.55 , the entropy production becomes increasingly positive with increasing coupling and tends asymptotically to a line with slope $\sim N^{2}$ at strong coupling. This finite, positive entropy production arises exactly when a fraction of oscillators in the network gets synchronized through the coupling. It represents the price that we have to pay for getting this ordered phase stabilized in time. For coupling parameter below the critical value, the network is disordered and there is no dissipation (i.e. the entropy production vanishes).

When all natural frequencies of the oscillators are equal we can prove analytically that the entropy production increases in driving the system to the stationary state. In this case we have $\omega_{i}^{\circ}=\omega^{\circ}$ for all $i$, and we can change the angle variables to $\phi_{i}=\theta_{i}-\omega^{\circ} t$. Eqs. (32) read in this case as

$$
\omega_{i}=\frac{\partial H}{\partial J_{i}}=K \sum_{j \neq i}^{N} \sin \left(\phi_{j}-\phi_{i}\right)
$$

and the entropy production, Eq. (34), is

$$
\frac{\sigma}{k}=K \sum_{j \neq i}^{N} \sum_{i}^{N} \cos \left(\phi_{j}-\phi_{i}\right)
$$


By comparing Eqs.(35) and (36) we observe that

$$
\omega_{i}=\frac{1}{k}\left(\frac{\partial \sigma}{\partial \phi_{i}}\right)
$$

and therefore

$$
\frac{d \sigma}{d t}=\sum_{i}^{N}\left(\frac{\partial \sigma}{\partial \phi_{i}}\right) \omega_{i}=\frac{1}{k} \sum_{i}^{N}\left(\frac{\partial \sigma}{\partial \phi_{i}}\right)^{2} \geq 0
$$

which is the result that we wanted to prove.

\section{CONCLUSIONS AND PERSPECTIVES}

We have shown that thermodynamics can be grounded on classical mechanics with the help of the Maupertuis principle. In our approach, thermal states are fully characterized by appropriate action variables. The nonintegrable part of the Hamiltonian dictates relaxation to equilibrium, an evolution driven to an steady state or a departure from equilibrium. This approach is consistent with the Second Law of Thermodynamics for macroscopic systems. The results obtained are, therefore, quite general and can be applied to systems with arbitrary size both in and out of equilibrium. Although we do not make explicit use of probability, it is also shown that the latter can be introduced from classical mechanics yielding an Einstein relationship at the thermodynamic limit. The main difficulty in our approach comes from finding the appropriate set of action-angle variables from a given Hamiltonian. We have shown, however, that in many relevant cases this is possible provided that a criterion on the suitability of these variables (coming from demanding a necessary consistency for the definition of the nonequilibrium entropy) is satisfied. Our approach has then been illustrated by applying it to systems with very different physical properties (ideal and globally chaotic systems and many-body Hamiltonians with short and long-range interactions). The dynamical definitions of thermodynamic quantities reproduce previous results derived from statistical mechanics. Finally, the relevance and applicability of our ideas to nonequilibrium situations has been illustrated by evaluating the (dynamically-defined) entropy production of a network of coupled oscillators (Kuramoto model). This quantity proves to be physically meaningful giving a measure of dynamical order. We find that its positive value at the steady state is directly related to the emergence of an ordered, synchronized phase from the collective dynamics of the coupled oscillators. This opens the possibility of studying the thermodynamics of pattern recognition [27, 28].

We acknowledge fruitful conversations with Profs. Katharina Krischer, Miguel A. Sanchis-Lozano and Alejandro Casanovas. This work was supported by the MEC (Ministry of Education and Science of Spain) and FEDER under Project No. MAT2005-01441.

\section{APPENDIX A: ERGODICITY}

The thermodynamic formalism presented in this paper does not require ergodicity. However, it embodies ergodicity when Birkhoff's theorem holds. From this theorem (see [17] p. 286), time averages $\langle\ldots\rangle_{\tau_{c}}$ coincide with phase space averages $\langle\ldots\rangle_{\Gamma}$ when all frequencies $\omega_{i}=\partial H / \partial J_{i}$ are independent. In this case, Eq. (15) becomes the statement of the equipartition principle and the system is ergodic. We then have $f k T^{(e q)}=<$ $2 K>_{\tau_{c}}=<2 K>_{\Gamma}=f \Phi(E) / \Omega(E)$ [30] where $\Phi=$ $\int d \Gamma \Theta[E-H(\mathbf{q}, \mathbf{p})]$ and $\Omega=\int d \Gamma \delta[E-H(\mathbf{q}, \mathbf{p})] . \quad \Theta$ and $\delta$ are the Heaviside and the Dirac delta functions, respectively, and $d \Gamma$ is the phase space volume element. Since $\Omega=\partial \Phi / \partial E$, we find that $f k T^{(e q)}=$ $f(\partial \ln \Phi / \partial E)^{-1}$. Taking this expression to Eq. (7) we obtain $\left(J_{c}\right)^{f}=\Phi$ and $S^{(e q)}=k \ln \Phi$. This is the so-called (Hertz) volume entropy which is an adiabatic invariant 29, 31]. Birkhoff theorem does not hold, however, when there exists any integral relation between the frequencies $\omega_{i}$ [17]. In these cases, Eq. (15) can depart from ergodicity and equipartition.

\section{APPENDIX B: THE MIXING OF TWO IDEAL FLUIDS}

A popular topic in equilibrium thermodynamics is the mixing of two ideal fluids (see [8] for a beautiful explanation of Boltzmann ideas). Let us consider two ideal fluids at equilibrium mixed in a container closed by adiabatic rigid walls. The particles interact only through elastic collisions. This isolated system is depicted schematically in Fig. 4 (a) and is composed of pink and black particles. We ask now why we do not observe an spontaneous departure from this homogeneous mixture leading to a

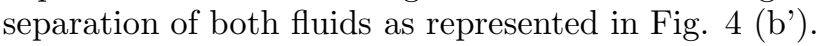

The Maupertuis principle requires that

$$
\delta J_{E}=\int_{\mathbf{q}_{0}}^{\mathbf{q}_{1}} \mathbf{p} d \mathbf{q}=\sqrt{2 m E} \delta \int_{\mathbf{q}_{0}}^{\mathbf{q}_{1}} d s=0
$$

where $d s$ is the differential of arc length on the trajectory. The last equality states that the trajectory of the motion between two points in the configuration space (say (a) and (c) in Fig. (4) is the one with minimal length. We now can immediately realize why we do not observe (b') starting from (a). The number of points in the trajectory in which the system looks macroscopically disordered as (a), (b) and (c) is large and points (a) and (c) are connected through points as (b) and not as ( $b$ '), because going through intermediate states of disorder as (b) extremizes the abbreviated action. It can be intuitively seen with the help of Fig. 4 that, from a mixed state (a), separating pink particles from black particles (b') and then mixing them again (c) leads to a longer trajectory than passing through intermediate mixed states such as (b). Attaining (b') from (a) requires each particle to travel a 


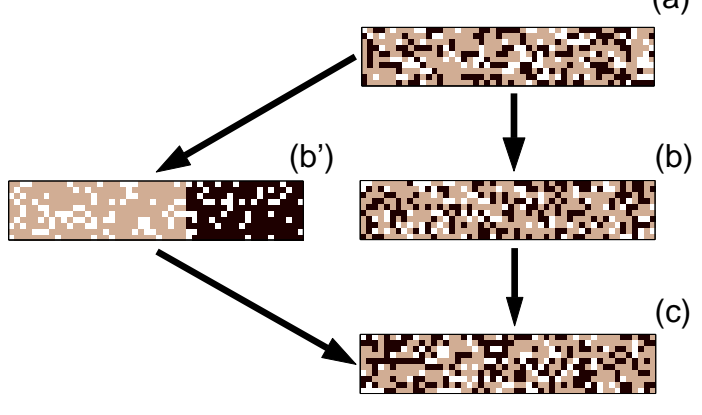

FIG. 4: Two possible trajectories joining the disordered configurations (a) and (c) of two mixed fluids. The trajectory going through (b') is forbidden by the Maupertuis principle since it is possible to find disordered configurations (b) between (a) and (c) so that the abbreviated action (三 the length of the trajectory between (a) and (c) in the $3 \mathrm{~N}$-dimensional configuration space) is a minimum.

certain distance so that it is then located in the appropriate macroscopic subvolume. This requirement is not necessary if the trajectory passes through intermediate disordered states such as (b). Each individual particle travels now a much shorter distance and so is the total length of the trajectory in the $3 \mathrm{~N}$-space. By Maupertuis principle, the shortest path is the one chosen by the motion (in the typical time interval of a measurement) so that passing through (b') is forbidden.

Although points like (b') lie far apart of the trajectory of the motion, the above considerations do not rule out totally the possibility of attaining them. Some of these points can belong to the whole closed trajectory but lie in segments which have a total duration which is very short compared to the whole closed trajectory. These segments are not the typical ones of the trajectory. The typical segments, whose properties contribute most significantly to the time average on the closed trajectory join points as (a)-(b)-(c).

All above considerations can be easily extended to any Hamiltonian system at equilibrium but then the quantity equivalent to the minimum of the abbreviated action is not the minimal geometric length of the trajectory but the extremum of the quantity

$$
\int_{\mathbf{q}_{0}}^{\mathbf{q}_{1}} \sqrt{2 m(E-U)} d s
$$

The integrand $\sqrt{2 m(E-U)}$ plays here a similar role to the index of refraction in Fermat's principle of the minimum optical path.
[1] C. G. Gray, G. Karl, V. A. Novikov, Rep. Prog. Phys. 67 (2004) 159.

[2] L. D. Landau, E. M. Lifshitz, Mechanics (Pergamon Press, Oxford, 1982).

[3] L. Casetti, M. Pettini, E. G. D. Cohen, Phys. Rep. 337 (2000) 238.

[4] M. Bailyn, A Survey of Thermodynamics (American Institute of Physics Press, New York, 1994), Chapter 10.

[5] G. Gallavotti, Statistical Mechanics. A short treatise (Springer Verlag, Berlin, 1999).

[6] M. Campisi, Stud. Hist. Philos. Mod. Phys. 36 (2005) 275.

[7] J. L. Lebowitz, Physica A 263 (1999) 516.

[8] J. L. Lebowitz, Phys. Today. 46 (1993) 32.

[9] E. G. D. Cohen, Pramana-J. Phys. 64 (2005) 635.

[10] D. H. E. Gross, Microcanonical Thermodynamics: Phase transitions in "Small" systems (World Scientific, Singapore, 2001); D. H. E. Gross, Phys. Chem. Chem. Phys. 4 (2002) 863.

[11] G. Gallavotti, J. Stat. Phys. 78 (1995) 1571.

[12] E. G. D. Cohen, Physica A 305 (2002) 19.

[13] I. C. Percival, J. Phys. A. 7 (1974) 794; S. Chapman, B. C. Garrett, W. H. Miller J. Chem. Phys. 64 (1976) 502.

[14] M. Kaasalainen, Phys. Rev. E 52 (1995) 1193; M. Kaasalainen and J. Binney, Phys. Rev. Lett. 73 (1994) 2377.

[15] L. Boltzmann, Wien Ber. 53 (1866) 195.

[16] P. J. Morrison, Rev. Mod. Phys. 70 (1998) 467.

[17] V. I. Arnold, Mathematical Methods of Classical Mechan- ics (Springer Verlag, New York, 1978).

[18] V. Garcia-Morales, J. Pellicer, Physica A 361 (2006) 161.

[19] P. T. Landsberg, Thermodynamics and Statistical Mechanics (Dover, New York, 1990).

[20] M. Abramowitz and I. A. Stegun, Handbook of Mathematical Functions (Dover, New York, 1972), p. 10.

[21] C. C. Martens, R. L. Waterland, W. P. Reinhardt, J. Chem. Phys. 90 (1989) 2328.

[22] V. Karimipour, J. Math. Phys. 38 (1997) 1577.

[23] Y. Kuramoto, Chemical Oscillations, Waves and Turbulence (Springer Series in Synergetics, Berlin, 1984).

[24] J. J. Acebrón, L. L. Bonilla, C. J. Pérez Vicente, F. Ritort, R. Spigler, Rev. Mod. Phys. 77 (2005) 137.

[25] S. H. Strogatz, Physica D 143 (2000) 1.

[26] A. S. Mikhailov, D. H. Zanette, Y. M. Zhai, I. Z. Kiss, J. L. Hudson, Proc. Nat. Acad. Sci. 101 (2004) 10890.

[27] F. C. Hoppensteadt, E. M. Izhikevich, Phys. Rev. Lett. 82 (1999) 2983.

[28] F. C. Hoppensteadt and E. M. Izhikevich, IEEE Trans. Neural Networks 11 (2000) 734.

[29] V. L. Berdichevsky, Thermodynamics of Chaos and Order (Longman, Edinburgh Gate, Harlow, England, 1997).

[30] A. I. Khinchin Mathematical foundations of statistical mechanics (NY, Dover, 1949) p. 107 Eq. (60).

[31] A. B. Adib, J. Stat. Phys. 117 (2004) 581; A. B. Adib, Phys. Rev. E 66 (2002) 047101. 